Naohiko Seki ${ }^{1} *$ Sumio Sugano • Yutaka Suzuki

Akira Nakagawara · Miki Ohira · Masa-aki Muramatsu

Toshiyuki Saito • Tada-aki Hori

\title{
Isolation, tissue expression, and chromosomal assignment of human RGS5, a novel G-protein signaling regulator gene
}

Received: April 17, 1998 / Accepted: May 28, 1998

\begin{abstract}
The regulator of G-protein signaling (RGS) proteins have recently been identified as signal transduction molecules which have structural homology to SST2 of Saccharomyces cerevisiae and EGL-10 of Caenorhabditis elegans. Multiple genes homologous to SST2 are present in higher eukaryotes, and the group of these genes is termed the RGS family. RGS proteins are involved in the regulation of heterotrimeric G-proteins by acting as GTPase-activators. A putative new member of the RGS family was isolated from a neuroblastoma cDNA library. The amino acid sequence deduced from the cDNA possessed all consensus motifs of the RGS domain and showed closest homology to mouse RGS5 (90\% identical), indicating that it was human RGS5 (hRGS5). The messenger RNA of $h R G S 5$ was abundantly expressed in heart, lung, skeletal muscle, and small intestine, and at low levels in brain, placenta, liver, colon, and leukocytes. The chromosome localization of the gene in the 1q23 region was determined by a monochromosomal hybrid panel and a radiation hybrid panel.
\end{abstract}

Key words G-protein · RGS5 - Chromosome mapping • 1q23 $\cdot$ Neuroblastoma $\cdot$ cDNA library

N. Seki $(\bowtie) \cdot$ T. Saito $\cdot$ T. Hori

Genome Research Group, National Institute of Radiological

Sciences, 4-9-1 Anagawa, Inage-ku, Chiba 263-8555, Japan

Tel. +81-43-206-3135; Fax +81-43-251-9818

e-mail: nseki@nirs.go.jp

S. Sugano $\cdot$ Y. Suzuki

Department of Virology, Institute of Medical Science, the University of Tokyo, Tokyo, Japan

A. Nakagawara $\cdot$ M. Ohira

Division of Biochemistry, Chiba Cancer Center, Chiba, Japan

M. Muramatsu

Helix Research Institute, Chiba, Japan and Department of Bioregulation, Medical Research Institute, Tokyo Medical Dental

University, Tokyo, Japan

\section{Introduction}

Heterotrimeric G-proteins comprise a superfamily which is involved in the transmission of extracellular signals of hormones and neurotransmitters across the cell membrane to regulate various physiological processes. Recently, novel regulatory molecules for these G-protein signaling pathways, termed RGS, have been identified (Koelle and Horvits 1996; Koelle 1997; Dohlman and Thorner 1997).

The function of the RGS family was initially discovered from the genetic analysis of two independent model organisms, Saccharomyces cerevisiae and Caenorhabditis elegans. In $S$. cerevisiae, SST2, the prototype for the newly recognized RGS family, is involved in the desensitization of mating pheromone, which operates through a heterotrimeric G-protein signalling pathway (Dohlman et al. 1996; Druey et al. 1996). Rat RGS 1-4 have been shown to partially complement a defect of SST2 in yeast, indicating an evolutional conservation of structure and function (Druey et al. 1996). Another line of functional evidence for RGS has emerged from studies of the neurotransmittermodulated egg-laying behavior of C. elegans. Goa-1, a Go alpha subunit regulates this behavior, and EGL-10, an RGS protein, regulates this behavior in an opposite manner, and genetic analysis has suggested that EGL-10 regulates Gprotein signaling pathways (Koelle and Horvits 1996). Further biochemical analysis of the RGS family has assigned their function as GTPase-activating proteins of heteromeric G-proteins (Berman et al. 1996; Chatterjee et al. 1997; Popov et al. 1997).

hRGS5 cDNA was cloned from a full-length enriched cDNA library constructed from a neuroblastoma (NB) sample using the oligo-capping method, as described previously (Maruyama and Sugano 1994; Suzuki et al. 1997). Here we report the sequence features, expression profile, and chromosomal assignment of the hRGS5 gene. This information should prove valuable in designing studies to evaluate its cellular function in G-protein signaling. 


\section{Materials and methods}

Isolation and sequencing of human RGS5

We constructed a full-length enriched cDNA library derived from the NB specimen, using the oligo-capping method (Maruyama and Sugano 1994; Suzuki et al. 1997). A cDNA clone revealed from one-pass sequence that it had closest homology to mouse and rat RGS5. The entire sequence of this clone was determined by a shot-gun strategy (Seki et al. 1997) and searches of the public databases were performed routinely with the FASTA program of the UWGCG package (Pearson and Lipman 1988).

\section{Chromosome mapping}

Chromosomal assignment of the $h R G S 5$ was done by polymerase chain reaction (PCR) analysis of human and rodent somatic cell hybrid panels. DNA of the human-rodent somatic cell hybrid panel (Mapping panel no. 2) and radiation hybrid panel (Genebridge 4) were purchased from the National Institute of General Medicine Service, Coriell Cell Repositories (Camden, NJ, USA) and Research Genetics (Huntsvill, AL, USA), respectively. Primers used for PCR amplification correspond to the nucleotide (n. t.) 1573 to n. t. 1593 (5'-TGGCTAATTGTCCTATGATGC-3') and the n. t. 1741 to n. t. 1761 (5'-GTGAAGGAATACTGGGGTTGC-3')(PCR product size, 189bp). PCR was carried out in the manner described previously (Saito et al. 1997; Seki et al. 1997).

\section{Northern blot analysis}

Northern blot analysis of hRGS5 mRNA in various human tissues. Northern blot filters containing adult human poly (A) ${ }^{+}$RNAs $(2 \mu \mathrm{g} /$ lane) were purchased from Clontech Laboratories (Palo Alto, CA, USA) and hybridization and washing were performed following the manufacturer's in- structions. The 2.1-kb cDNA fragment containing the entire open reading frame was labelled with $\left[\alpha-{ }^{32} \mathrm{P}\right] \mathrm{dCTP}$ and used as a hybridization probe.

\section{Results and discussion}

The isolated hRGS5 cDNA clone was 2076bp in length and had an open reading frame of 181 amino acids. The predicted protein of 181 amino acids had a calculated molecular weight of approximately $21-\mathrm{kDa}$. The nucleotide sequence data reported here will appear in the DDBJ, EMBL and GenBank nucleotide sequence databases with the accession number AB008109. A homology search of the conceptual translated amino acid sequence of the isolated cDNA revealed that it was most homologous to mouse RGS5 (Chen et al. 1997), having 90\% identity at the amino acid level, and thus we judged this clone was derived from the human RGS5 (hRGS5) gene. The alignment of the deduced amino acid sequences of hRGS5, mouse RGS5, and rat RGS5 (partial sequence) (Druey et al. 1996) is shown in Fig. 1.

We determined the chromosomal location of the RGS5 gene using a somatic cell hybrid panel. A PCR product of the expected size of $189 \mathrm{bp}$ was detected in human genomic DNA, control cDNA, and somatic cell hybrids containing only human chromosome 1 (data not shown). We performed PCR analysis of a radiation hybrid panel with the same primers as those used in the assay for the somatic cell hybrid panel. Statistical analysis of the radiation hybrid data was performed using the (RHMAPPER) software package (http://www-genome.wi.mit.edu/cgi-bin/contig/rhmapper. pl). The data vector for the $h R G S 5$ gene was 1001010001 $\begin{array}{llll}0010121210 & 0000010100 & 0011000010 & 1010101001\end{array}$ 0101000000001000000001011000000100000000021 , and the consequent report indicated the gene was mapped between markers GHCL.GATA70D01 and WI-4182, both of which have been cytogenetically mapped to $1 \mathrm{q} 23$. The position is $0.00 \mathrm{cR}$ from GHCL.GATA70D01 ( $\mathrm{lod}>3.0)$. To confirm the PCR-based chromosome mapping by an inde-

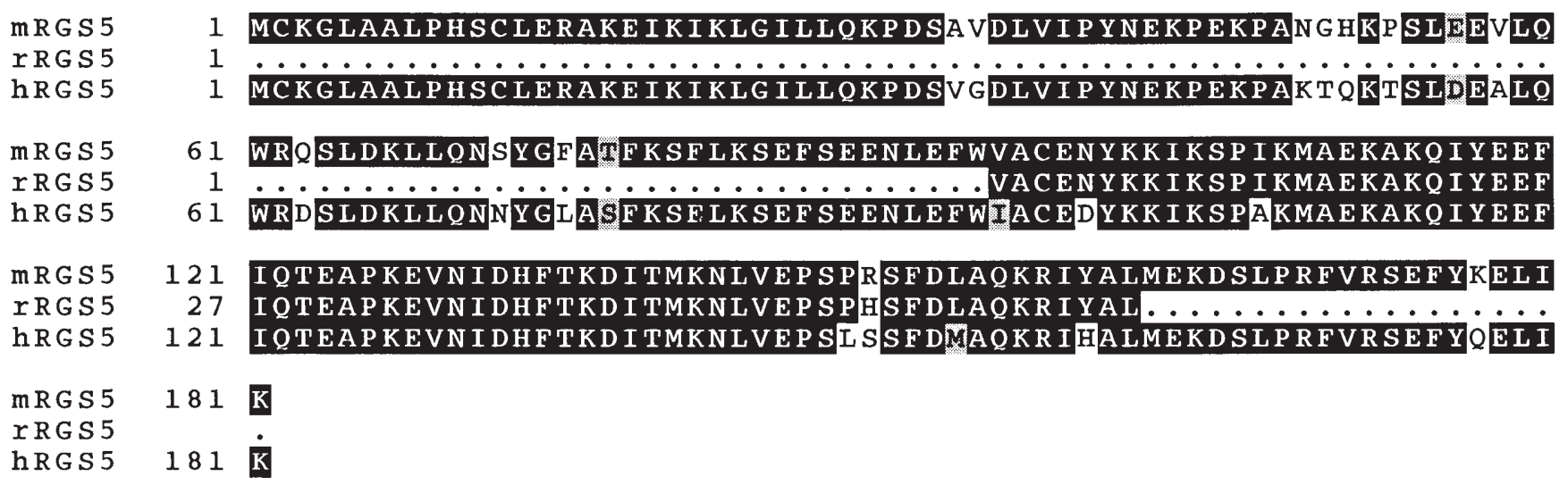

Fig. 1 Alignment of human ( $h$ ) RGS5 (accession no., AB008109), mouse ( $m$ ) RGS5 (accession no., U67188), and rat ( $r$ ) RGS5 (accession no., U32435). Identities are indicated by black background, and similar residues are shadowed 
Fig. 2 Northern blot analysis of human RGS5. Northern blot filters containing adult human poly $(\mathrm{A})^{+}$RNAs $(2 \mu \mathrm{g} /$ lane) were purchased from Clontech Laboratories (Palo Alto, CA, USA), and hybridization and washing were performed following the manufacturer's instructions. The 2.1-kb cDNA fragment containing the entire open reading frame was labelled with $\left[\alpha-{ }^{32} \mathrm{P}\right] \mathrm{dCTP}$ and used as a hybridization probe. The bottom panel shows the result with a $\beta$-actin probe used to adjust for the amount of RNA loaded. Size markers (left) are in kilobases $(k b)$

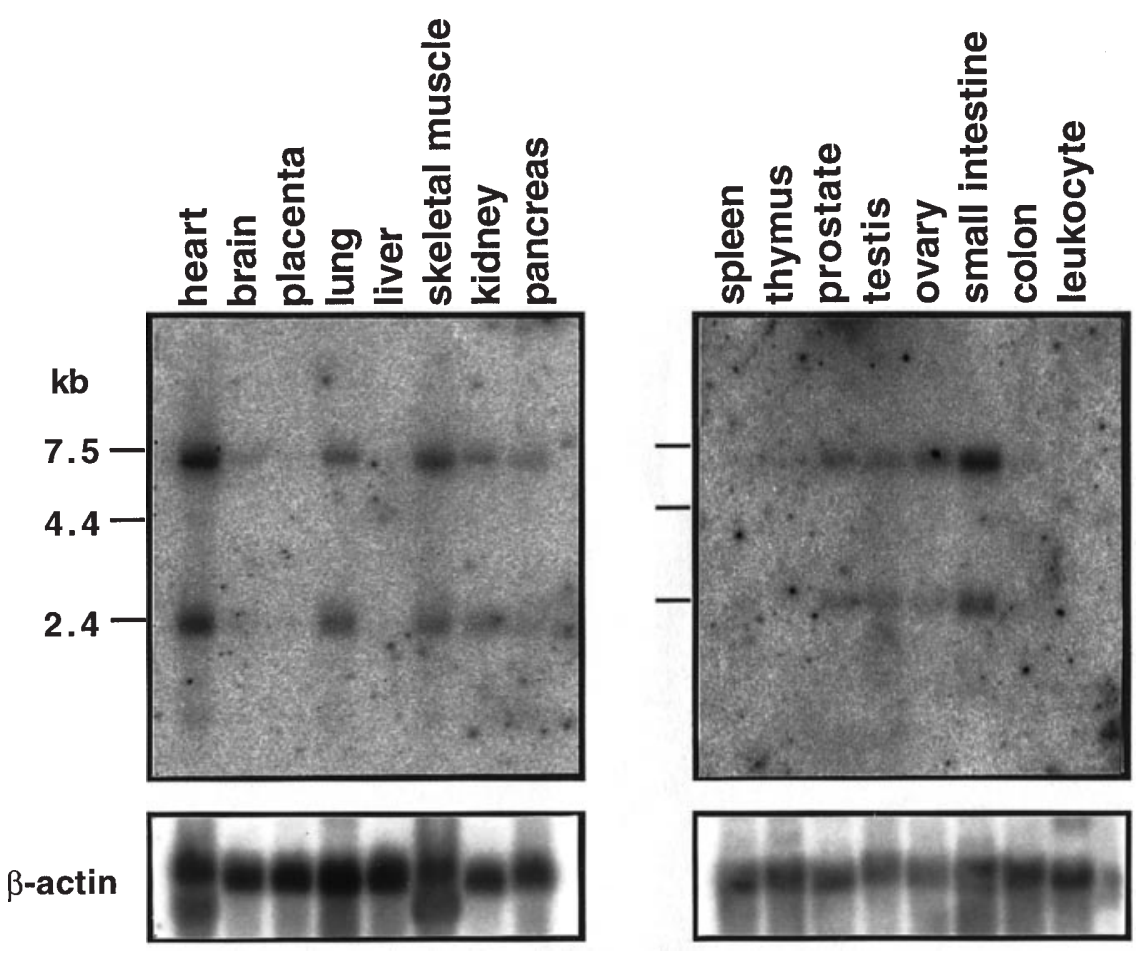

pendent approach, we performed R-banding fluorescence in-situ hybridization (FISH), using a P1 phage DNA, as described previously (Seki et al. 1997). Clear doublet signals were consistently demonstrated on the q23 region of chromosome 1 (data not shown). Therefore, the gene was mapped on the q23 region of chromosome 1 .

We examined the tissue distribution of RGS5 in various tissues by northern blot analysis. Hybridization of human RNAs from multiple tissues (Clontech) with a RGS5 cDNA probe detected two mRNA species, which migrated as 2.1-kb and 7.0-kb bands (Fig. 2). The size of the band is consistent with the notion that our clone is full length. The hRGS5 was abundantly expressed in heart, lung, skeletal muscle, and small intestine, and at low levels in brain, placenta, liver, colon, and leukocytes. These observations are similar to findings for mouse RGS5 (Chen et al. 1997). Recently, the localization of nine rat RGSs, including RGS5, was investigated systematically in rat brain, using an in-situ hybridization technique; the RGS5 mRNA was expressed at lower density in rat brain than in the other tissues examined (Gold et al. 1997).

A large number of RGSs have been identified through a database search for expressed sequence tags (ESTs) and screening by a PCR-assisted method (Druey et al. 1996; Koelle 1997). In addition a yeast two-hybrid system using Gai3 has identified GAIP (De Vries et al. 1995), another member of the RGS family (Berman 1996). Also recently identified is axin, a gene regulating the embryonic axis in the mouse, and this was found to have structural similarities with RGSs (Zeng et al. 1997).

RGS proteins can now be seen to be associated with a large gene family, with an ancestral, origin in lower eukaryotes; the number of members of the family is still rap- idly expanding. The physiological significance and biochemical properties of different RGS proteins remain to be explored.

\section{References}

Berman DM, Wilkie TM, Gilman AG (1996) GAIP and RGS4 are GTPase-activating proteins for the Gi subfamily of $G$ protein alpha subunits. Cell 86: 445-452

Chatterjee TK, Eapen AK, Fisher RA (1997) A truncated form of RGS3 negatively regulates $G$ protein-coupled receptor stimulation of adenylyl cyclase and phosphoinositide phospholipase C. J Biol Chem 272: 15481-15487

Chen C, Zheng B, Han J, Lin SC (1997) Characterization of a novel mammalian RGS protein that binds to $G$ alpha proteins and inhibits pheromone signaling in yeast. J Biol Chem 272: 8679-8685

De Vries L, Mousli M, Wurmser A, Farquhar MG (1995) GAIP, a protein that specifically interacts with the trimeric $G$ protein $G$ alpha $\mathrm{i} 3$, is a member of a protein family with a highly conserved core domain. Proc Natl Acad Sci USA 92: 11916-11920

Dohlman HG, Song J, Ma D, Courchesne WE, Thorner J (1996) Sst2, a negative regulator of pheromone signaling in the yeast Saccharomyces cerevisiae: Expression, localization, and genetic interaction and physical association with Gpa1 (the G-protein alpha subunit). Mol Cell Biol 16: 5194-5209

Dohlman HG, Thorner J (1997) RGS proteins and signaling by heterotrimeric $\mathrm{G}$ proteins. J Biol Chem 14: 3871-3874

Druey KM, Blumer KJ, Kang VH, Kehrl JH (1996) Inhibition of Gprotein mediated MAP kinase activation by a new mammalian gene family. Nature 379: 742-746

Gold SJ, Ni YG, Dohlman HG, Nestler EJ (1997) Regulators of Gprotein signaling (RGS) proteins: Region-specific expression of nine subtypes in rat brain. J Neurosci 17: 8024-8037

Koelle MR, Horvitz HR (1996) EGL-10 regulates G protein signaling in the $C$. elegans nervous system and shares a conserved domain with many mammalian proteins. Cell 84: $115-125$

Koelle MR (1997) A new family of G-protein regulators - the RGS proteins. Curr Opin Cell Biol 9: 143-147 
Maruyama K, Sugano S (1994) Oligo-capping: A simple method to replace the cap structure of eukaryotic mRNAs with oligoribonucleotides. Gene 138: 171-174

Pearson WR, Lipman DJ (1988) Improved tools for biological sequence comparison. Proc Natl Acad Sci USA 85: 2444-2448

Popov S, Yu K, Kozasa T, Wilkie TM (1997) The regulation of Gprotein signaling (RGS) domains of RGS4, RGS10, and GAIP retain GTPase activating protein activity in vitro. Proc Natl Acad Sci USA 94: 7216-7220

Saito T, Seki N, Ishii H, Ohira M, Hayashi A, Kozuma S, Hori T (1997) Complementary DNA cloning and chromosomal mapping of a novel phosphatidylinositol kinase gene. DNA Res 4: 301-305
Seki N, Nimura Y, Ohira M, Saito T, Ichimiya S, Nomura N, Nakagawara A (1997) Identification and chromosome assignment of a human gene encoding a novel phosphatidylinositol-3 kinase. DNA Res 4: 355-358

Suzuki Y, Yoshitomo-Nakagawa K, Maruyama K, Suyama A, Sugano S (1997) Construction and characterization of a full-enriched length and a 5'-end-enriched cDNA library. Gene 200: 149-156

Zeng L, Fagotto F, Zhang T, Hsu W, Vasicek TJ, Perry WL 3rd, Lee JJ, Tilghman SM, Gumbiner BM, Costantini F (1997) The mouse Fused locus encodes Axin, an inhibitor of Wnt signaling pathway that regulates embryonic axis formation. Cell 90: 181-192 